\title{
Sequence of Numbers with Three Alternate Common Differences and Common Ratios
}

\author{
Julius Fergy T. Rabago \\ Faculty, Department of Mathematics and Physics \\ College of Arts and Sciences, Central Luzon State University \\ Science City of Muñoz 3120, Nueva Ecija, Philippines \\ Email: julius_fergy.rabago@up.edu.ph
}

\begin{abstract}
This paper talks about two types of special sequences. The first is the arithmetic sequence of numbers with three alternate common differences; and the other, is the geometric sequence of numbers with three alternate common ratios. The formulas for the general term $a_{n}$ and the sum of the first $n$ terms, denoted by $S_{n}$, are given respectively.
\end{abstract}

Keywords: Sequence of numbers with three alternate common differences, sequence of numbers with three alternate common ratios, general term $a_{n}$, the sum of the first $n$ terms denoted by $S_{n}$.

\section{Arithmetic sequence of numbers with three alternate common differences}

Definition 1.1. A sequence of numbers $\left\{a_{n}\right\}$ is called a sequence of numbers with three alternating common differences if the following conditions are satisfied:

(i) for all $k \in N, a_{3 k-1}-a_{3 k-2}=d_{1}$,

(ii) for all $k \in N, a_{3 k}-a_{3 k-1}=d_{2}$,

(iii) for all $k \in N, a_{3 k+1}-a_{3 k}=d_{3}$,

here $d_{1}\left(d_{2}\right.$, and $\left.d_{3}\right)$ is called the first (the second and the third) common differences of $\left\{a_{n}\right\}$. 
Example 1.2. The number sequence 1, 2, 4, 7, 8, 10, 13, 14, 16, .. is a sequence of numbers with three alternate common differences, where $d_{1}=$ $1, d_{2}=2$, and $d_{3}=3$.

Obviously, $\left\{a_{n}\right\}$ has the following form

$$
\begin{gathered}
a_{1}, a_{1}+d_{1}, a_{1}+d_{1}+d_{2}, a_{1}+d_{1}+d_{2}+d_{3}, a_{1}+2 d_{1}+d_{2}+d_{3}, a_{1}+2 d_{1}+2 d_{2}+d_{3}, \\
a_{1}+2 d_{1}+2 d_{2}+2 d_{3}, a_{1}+3 d_{1}+2 d_{2}+2 d_{3}, a_{1}+3 d_{1}+3 d_{2}+2 d_{3}, \ldots
\end{gathered}
$$

Theorem 1.3. The formula of the general term of $a_{n}$ is

$$
a_{n}=a_{1}+\left\lfloor\frac{n+1}{3}\right\rfloor d_{1}+\left\lfloor\frac{n}{3}\right\rfloor d_{2}+\left\lfloor\frac{n-1}{3}\right\rfloor d_{3}
$$

Proof. We prove this theorem by induction on $n$.

Obviously, (1) holds for $n=1,2,3$ and 4 .

Suppose (1) holds when $n=k$, hence

$$
a_{k}=a_{1}+\left\lfloor\frac{k+1}{3}\right\rfloor d_{1}+\left\lfloor\frac{k}{3}\right\rfloor d_{2}+\left\lfloor\frac{k-1}{3}\right\rfloor d_{3}
$$

We need to show that $\mathrm{P}(k+1)$ also holds for any $k \in N$.

(i.) If $k=3 m-2$, where $m \in N$, then $a_{k+1}=a_{k}+d_{1}$

$$
\begin{aligned}
a_{k+1} & =a_{1}+\left\lfloor\frac{k+1}{3}\right\rfloor d_{1}+\left\lfloor\frac{k}{3}\right\rfloor d_{2}+\left\lfloor\frac{k-1}{3}\right\rfloor d_{3}+d_{1} \\
& =a_{1}+\left\lfloor\frac{3 m-2+1}{3}\right\rfloor d_{1}+\left\lfloor\frac{3 m-2}{3}\right\rfloor d_{2}+\left\lfloor\frac{3 m-2-1}{3}\right\rfloor d_{3}+d_{1} \\
& =a_{1}+(m-1) d_{1}+(m-1) d_{2}+(m-1) d_{3}+d_{1} \\
& =a_{1}+\left\lfloor\frac{3 m}{3}\right\rfloor d_{1}+\left\lfloor m-1+\frac{2}{3}\right\rfloor d_{2}+\left\lfloor m-1+\frac{1}{3}\right\rfloor d_{3} \\
& =a_{1}+\left\lfloor\frac{(k+1)+1}{3}\right\rfloor d_{1}+\left\lfloor\frac{k+1}{3}\right\rfloor d_{2}+\left\lfloor\frac{(k+1)-1}{3}\right\rfloor d_{3}
\end{aligned}
$$

$\therefore \mathrm{P}(k+1)$ holds for $k=3 m-2$.

(ii.) If $k=3 m-1$, where $m \in N$, then $a_{k+1}=a_{k}+d_{2}$

$$
\begin{aligned}
a_{k+1} & =a_{1}+\left\lfloor\frac{k+1}{3}\right\rfloor d_{1}+\left\lfloor\frac{k}{3}\right\rfloor d_{2}+\left\lfloor\frac{k-1}{3}\right\rfloor d_{3}+d_{2} \\
& =a_{1}+\left\lfloor\frac{3 m-1+1}{3}\right\rfloor d_{1}+\left\lfloor\frac{3 m-1}{3}\right\rfloor d_{2}+\left\lfloor\frac{3 m-1-1}{3}\right\rfloor d_{3}+d_{2}
\end{aligned}
$$




$$
\begin{aligned}
& =a_{1}+m d_{1}+(m-1) d_{2}+(m-1) d_{3}+d_{2} \\
& =a_{1}+\left\lfloor m+\frac{1}{3}\right\rfloor d_{1}+\left\lfloor\frac{3 m}{3}\right\rfloor d_{2}+\left\lfloor m-1+\frac{2}{3}\right\rfloor d_{3} \\
& =a_{1}+\left\lfloor\frac{(k+1)+1}{3}\right\rfloor d_{1}+\left\lfloor\frac{k+1}{3}\right\rfloor d_{2}+\left\lfloor\frac{(k+1)-1}{3}\right\rfloor d_{3}
\end{aligned}
$$

$\therefore \mathrm{P}(k+1)$ holds for $k=3 m-1$.

(iii.) If $k=3 m$, where $m \in N$, then $a_{k+1}=a_{k}+d_{3}$

$$
\begin{aligned}
a_{k+1} & =a_{1}+\left\lfloor\frac{k+1}{3}\right\rfloor d_{1}+\left\lfloor\frac{k}{3}\right\rfloor d_{2}+\left\lfloor\frac{k-1}{3}\right\rfloor d_{3}+d_{3} \\
& =a_{1}+\left\lfloor\frac{3 m+1}{3}\right\rfloor d_{1}+\left\lfloor\frac{3 m}{3}\right\rfloor d_{2}+\left\lfloor\frac{3 m-1}{3}\right\rfloor d_{3}+d_{3} \\
& =a_{1}+m d_{1}+m d_{2}+(m-1) d_{3}+d_{3} \\
& =a_{1}+\left\lfloor m+\frac{2}{3}\right\rfloor d_{1}+\left\lfloor m+\frac{1}{3}\right\rfloor d_{2}+\left\lfloor\frac{3 m}{3}\right\rfloor d_{3} \\
& =a_{1}+\left\lfloor\frac{(k+1)+1}{3}\right\rfloor d_{1}+\left\lfloor\frac{k+1}{3}\right\rfloor d_{2}+\left\lfloor\frac{(k+1)-1}{3}\right\rfloor d_{3}
\end{aligned}
$$

$\therefore \mathrm{P}(k+1)$ holds for $k=3 m$.

Therefore, (1) holds when $n=k+1$. This proves the theorem.

Theorem 1.4. The formula of the general term of $a_{n}$ can also be

$$
a_{n}=a_{1}+\left\lfloor\frac{n-1}{3}\right\rfloor d+\left(\left\lfloor\frac{n+1}{3}\right\rfloor-\left\lfloor\frac{n-1}{3}\right\rfloor\right) d_{1}+\left(\left\lfloor\frac{n}{3}\right\rfloor-\left\lfloor\frac{n-1}{3}\right\rfloor\right) d_{2}
$$

where $d=d_{1}+d_{2}+d_{3}$.

Formula (2) can be shown easily using induction on $n$. The proof for the theorem is ommited.

Now we proceed to the sum of the first $n$ terms of the sequence.

Theorem 1.5. The sum of the of the first $n$ terms of the sequence, denoted by $S_{n}$, is given by

$$
S_{n}=n a_{1}+\frac{1}{2} d \sum_{i=0}^{2}\left\lfloor\frac{n+i}{3}\right\rfloor+2\left(\left\lfloor\frac{n+1}{3}\right\rfloor d_{1}-\left\lfloor\frac{n}{3}\right\rfloor d_{3}\right)
$$

where $d=d_{1}+d_{2}+d_{3}$ 
Proof. Let $d=d_{1}+d_{2}+d_{3}$.

$$
\begin{aligned}
& S_{n}=a_{1}+\left(a_{1}+d_{1}\right)+\left(a_{1}+d_{1}+d_{2}\right)+\left(a_{1}+d_{1}+d_{2}+d_{3}\right) \\
& +\left(a_{1}+2 d_{1}+d_{2}+d_{3}\right)+\left(a_{1}+2 d_{1}+2 d_{2}+d_{3}\right)+\ldots \\
& +\left(a_{1}+\left\lfloor\frac{k+1}{3}\right\rfloor d_{1}+\left\lfloor\frac{k}{3}\right\rfloor d_{2}+\left\lfloor\frac{k-1}{3}\right\rfloor d_{3}\right) \\
& =\left(a_{1}+(1-1) d\right)+\left(a_{1}+d_{1}+(1-1) d\right)+\left(a_{1}+d_{1}+d_{2}+(1-1) d\right) \\
& +\left(a_{1}+(2-1) d\right)+\left(a_{1}+d_{1}+(2-1) d\right)+\left(a_{1}+d_{1}+d_{2}+(2-1) d\right) \\
& +\left(a_{1}+(3-1) d\right)+\ldots+\left(a_{1}+d_{1}+d_{2}+\left(\left\lfloor\frac{n}{3}\right\rfloor-1\right) d\right) \\
& +\left(a_{1}+d_{1}+\left(\left\lfloor\frac{n+1}{3}\right\rfloor-1\right) d\right)+\left(a_{1}+\left(\left\lfloor\frac{n+2}{3}\right\rfloor-1\right) d\right) \\
& =\left(\left\lfloor\frac{n+2}{3}\right\rfloor+\left\lfloor\frac{n+1}{3}\right\rfloor+\left\lfloor\frac{n}{3}\right\rfloor\right) a_{1}+\frac{1}{2}\left\lfloor\frac{n+2}{3}\right\rfloor\left(\left\lfloor\frac{n+2}{3}\right\rfloor-1\right) d \\
& +\left\lfloor\frac{n+1}{3}\right\rfloor d_{1}+\frac{1}{2}\left\lfloor\frac{n+1}{3}\right\rfloor\left(\left\lfloor\frac{n+1}{3}\right\rfloor-1\right) d+\left\lfloor\frac{n}{3}\right\rfloor\left(d_{1}+d_{2}\right) \\
& +\frac{1}{2}\left\lfloor\frac{n}{3}\right\rfloor\left(\left\lfloor\frac{n}{3}\right\rfloor-1\right) d \\
& =n a_{1}+\frac{1}{2}\left(\left\lfloor\frac{n+2}{3}\right\rfloor\left(\left\lfloor\frac{n+2}{3}\right\rfloor-1\right)+\left\lfloor\frac{n+1}{3}\right\rfloor\left(\left\lfloor\frac{n+1}{3}\right\rfloor-1\right)\right) d_{1} \\
& +\frac{1}{2}\left(2\left\lfloor\frac{n+1}{3}\right\rfloor+2\left\lfloor\frac{n}{3}\right\rfloor+\left\lfloor\frac{n}{3}\right\rfloor\left(\left\lfloor\frac{n}{3}\right\rfloor-1\right)\right) d_{1} \\
& +\frac{1}{2}\left(\left\lfloor\frac{n+2}{3}\right\rfloor\left(\left\lfloor\frac{n+2}{3}\right\rfloor-1\right)+\left\lfloor\frac{n+1}{3}\right\rfloor\left(\left\lfloor\frac{n+1}{3}\right\rfloor-1\right)\right) d_{2} \\
& +\frac{1}{2}\left(\left\lfloor\frac{n+2}{3}\right\rfloor\left(\left\lfloor\frac{n+2}{3}\right\rfloor-1\right)+\left\lfloor\frac{n+1}{3}\right\rfloor\left(\left\lfloor\frac{n+1}{3}\right\rfloor-1\right)\right) d_{3} \\
& +\frac{1}{2}\left(2\left\lfloor\frac{n}{3}\right\rfloor+\left\lfloor\frac{n}{3}\right\rfloor\left(\left\lfloor\frac{n}{3}\right\rfloor-1\right)\right) d_{2}+\frac{1}{2}\left(\left\lfloor\frac{n}{3}\right\rfloor\left(\left\lfloor\frac{n}{3}\right\rfloor-1\right)\right) d_{3} \\
& =n a_{1}+\frac{1}{2}\left(\left\lfloor\frac{n+2}{3}\right\rfloor\left\lfloor\frac{n-1}{3}\right\rfloor+\left\lfloor\frac{n+1}{3}\right\rfloor\left\lfloor\frac{n+4}{3}\right\rfloor+\left\lfloor\frac{n}{3}\right\rfloor\left\lfloor\frac{n+3}{3}\right\rfloor\right) d_{1} \\
& +\frac{1}{2}\left(\left\lfloor\frac{n+2}{3}\right\rfloor\left\lfloor\frac{n-1}{3}\right\rfloor+\left\lfloor\frac{n+1}{3}\right\rfloor\left\lfloor\frac{n-2}{3}\right\rfloor+\left\lfloor\frac{n}{3}\right\rfloor\left\lfloor\frac{n+3}{3}\right\rfloor\right) d_{2} \\
& +\frac{1}{2}\left(\left\lfloor\frac{n+2}{3}\right\rfloor\left\lfloor\frac{n-1}{3}\right\rfloor+\left\lfloor\frac{n+1}{3}\right\rfloor\left\lfloor\frac{n-2}{3}\right\rfloor+\left\lfloor\frac{n}{3}\right\rfloor\left\lfloor\frac{n-3}{3}\right\rfloor\right) d_{3} \\
& =n a_{1}+\frac{1}{2}\left(\left\lfloor\frac{n+2}{3}\right\rfloor\left(\left\lfloor\frac{n+2}{3}\right\rfloor-1\right)+\left\lfloor\frac{n+1}{3}\right\rfloor\left(\left\lfloor\frac{n+1}{3}\right\rfloor-1\right)\right) d \\
& +\frac{1}{2}\left\lfloor\frac{n}{3}\right\rfloor\left(\left\lfloor\frac{n}{3}\right\rfloor-1\right) d+2\left(\left\lfloor\frac{n+1}{3}\right\rfloor d_{1}-\left\lfloor\frac{n}{3}\right\rfloor d_{3}\right)
\end{aligned}
$$


Lemma 1.6. For any positive integers $p$, $q$, and $n$,

$$
\left[\frac{p}{q}\right]+n=\left[\frac{p+n q}{q}\right]
$$

Proof.

$$
\begin{aligned}
{\left[\frac{p}{q}\right] \quad } & \Rightarrow k \leq \frac{p}{q}<k+1 \text { where } k \text { is an integer } \\
& \Rightarrow m \leq \frac{p}{q}+n<m+1, m=n+k . \\
& \therefore \quad\left[\frac{p}{q}\right]+n=\left[\frac{p+n q}{q}\right] .
\end{aligned}
$$

Theorem 1.7. For any integer $m>0$

$$
\sum_{i=m q}^{n}\left\lfloor\frac{i}{m}\right\rfloor=\left\lfloor\frac{n}{m}\right\rfloor\left(n+1-m\left\lfloor\frac{n}{m}\right\rfloor\right)
$$

where $q=\left\lfloor\frac{n}{m}\right\rfloor$.

Proof.

$$
\begin{aligned}
\sum_{i=m q}^{n}\left\lfloor\frac{i}{m}\right\rfloor & =\sum_{i=0}^{n-m q}\left\lfloor\frac{i+m q}{m}\right\rfloor \\
& =\sum_{i=0}^{n-m q}\left(q+\left\lfloor\frac{i}{m}\right\rfloor\right) \\
& =\sum_{i=0}^{n-m q}\left\lfloor\frac{i}{m}\right\rfloor+\sum_{i=0}^{n-m q} q \\
& =\left\lfloor\frac{0}{m}\right\rfloor+\left\lfloor\frac{1}{m}\right\rfloor+\ldots+\left\lfloor\frac{n-m q}{m}\right\rfloor+q(n+1-m q) \\
& =\left\lfloor\frac{0}{m}\right\rfloor+\left\lfloor\frac{1}{m}\right\rfloor+\ldots+\left\lfloor\frac{n}{m}\right\rfloor-q+q(n+1-m q) \\
& =\left\lfloor\frac{n}{m}\right\rfloor\left(n+1-m\left\lfloor\frac{n}{m}\right\rfloor\right)
\end{aligned}
$$

Corollary 1.8. For any integer $m>0$,

$$
\sum_{i=0}^{n}\left\lfloor\frac{i}{m}\right\rfloor=\left\lfloor\frac{n}{m}\right\rfloor\left(n+1-\frac{m}{2}\left\lfloor\frac{n+m}{m}\right\rfloor\right)
$$


Proof. Let $q=\left\lfloor\frac{n}{m}\right\rfloor$

$$
\begin{aligned}
\sum_{i=0}^{n}\left\lfloor\frac{i}{m}\right\rfloor= & \sum_{i=0}^{m-1}\left\lfloor\frac{i}{m}\right\rfloor+\sum_{i=m}^{2 m-1}\left\lfloor\frac{i}{m}\right\rfloor+\ldots \\
& +\sum_{i=m(q-1)}^{m q-1}\left\lfloor\frac{i}{m}\right\rfloor+\sum_{i=m q}^{n}\left\lfloor\frac{i}{m}\right\rfloor \\
= & \sum_{j=0}^{q-1}\left(\sum_{i=j m}^{(j+1) m-1}\left\lfloor\frac{i}{m}\right\rfloor\right)+\sum_{i=m q}^{n}\left\lfloor\frac{i}{m}\right\rfloor \\
= & \sum_{j=0}^{q-1} m j+\sum_{i=m q}^{n}\left\lfloor\frac{i}{m}\right\rfloor \\
= & \frac{m q}{2}(q-1)+q(n+1-m q) \\
= & q\left(\frac{m q}{2}-\frac{m}{2}+n+1-m q\right) \\
= & \left\lfloor\frac{n}{m}\right\rfloor\left(n+1-\frac{m}{2}\left\lfloor\frac{n+m}{m}\right\rfloor\right)
\end{aligned}
$$

Theorem 1.9. The sum of the first $n$ terms of the sequence can also be

$$
\begin{aligned}
& S_{n}=n a_{1}+\left\lfloor\frac{n+1}{3}\right\rfloor\left(n+2-\frac{3}{2}\left\lfloor\frac{n+4}{3}\right\rfloor\right) d_{1}+\left\lfloor\frac{n}{3}\right\rfloor\left(n+1-\frac{3}{2}\left\lfloor\frac{n+3}{3}\right\rfloor\right) d_{2} \\
& +\left\lfloor\frac{n-1}{3}\right\rfloor\left(n-\frac{3}{2}\left\lfloor\frac{n+2}{3}\right\rfloor\right) d_{3}
\end{aligned}
$$

Proof.

$$
\begin{aligned}
S_{n}= & \sum_{i=1}^{n}\left(a_{1}+\left\lfloor\frac{i+1}{3}\right\rfloor d_{1}+\left\lfloor\frac{i}{3}\right\rfloor d_{2}+\left\lfloor\frac{i-1}{3}\right\rfloor d_{3}\right) \\
= & n a_{1}+\sum_{i=1}^{n}\left\lfloor\frac{i+1}{3}\right\rfloor d_{1}+\sum_{i=1}^{n}\left\lfloor\frac{i}{3}\right\rfloor d_{2}+\sum_{i=1}^{n}\left\lfloor\frac{i-1}{3}\right\rfloor d_{3} \\
= & n a_{1}+\left\lfloor\frac{n+1}{3}\right\rfloor\left(n+2-\frac{3}{2}\left\lfloor\frac{n+4}{3}\right\rfloor\right) d_{1} \\
& +\left\lfloor\frac{n}{3}\right\rfloor\left(n+1-\frac{3}{2}\left\lfloor\frac{n+3}{3}\right\rfloor\right) d_{2}+\left\lfloor\frac{n-1}{3}\right\rfloor\left(n-\frac{3}{2}\left\lfloor\frac{n+2}{3}\right\rfloor\right) d_{3}
\end{aligned}
$$




\section{Geometric sequence of numbers with three alternate common ratios}

Definition 2.1. A sequence of numbers $\left\{a_{n}\right\}$ is called a sequence of numbers with three alternating common ratios if the following conditions are satisfied:

(i) for all $k \in N, \frac{a_{3 k-1}}{a_{3 k-2}}=r_{1}$,

(ii) for all $k \in N, \frac{a_{3 k}}{a_{3 k-1}}=r_{2}$,

(iii) for all $k \in N, \frac{a_{3 k+1}}{a_{3 k}}=r_{3}$,

where $r_{1}, r_{2}$, and $r_{3}$ are called the first, the second and the third common ratios of $\left\{a_{n}\right\}$ respectively.

Example 2.2. The number sequence 1, 1/2, 1/6, 1/24, 1/48, 1/144, 1/576, $1 / 1152,1 / 3456, \ldots$ is an example of the sequence where $r_{1}=1 / 2, r_{2}=1 / 3$, and $r_{3}=1 / 4$.

Obviously, $\left\{a_{n}\right\}$ has the following form

$$
a_{1}, a_{1} r_{1}, a_{1} r_{1} r_{2}, a_{1} r_{1} r_{2} r_{3}, a_{1} r_{1}^{2} r_{2} r_{3}, a_{1} r_{1}^{2} r_{2}^{2} r_{3}, a_{1} r_{1}^{2} r_{2}^{2} r_{3}^{2}, a_{1} r_{1}^{3} r_{2}^{2} r_{3}^{2}, \ldots
$$

Theorem 2.3. The formula of the general term of $a_{n}$ is

$$
a_{n}=a_{1} \cdot r_{1}^{e_{n+1}} \cdot r_{2}^{e_{n}} \cdot r_{3}^{e_{n-1}}
$$

where $e_{i}=\left\lfloor\frac{i}{3}\right\rfloor$.

Proof. Let $e_{i}=\left\lfloor\frac{i}{3}\right\rfloor$ and use induction on $n$ to prove theorem 2.3.

Obviously, (3) holds for $n=1,2,3$ and 4 .

Now suppose (3) holds when $n=k$, hence

$$
a_{k}=a_{1} \cdot r_{1}^{e_{k+1}} \cdot r_{2}^{e_{k}} \cdot r_{3}^{e_{k-1}}
$$

We need to show that $\mathrm{P}(k+1)$ also holds for any $k \in N$.

(i.) If $k=3 m-2$, where $m \in N$, then $a_{k+1}=a_{k} \cdot r_{1}$ 


$$
\begin{aligned}
a_{k} & =a_{1} \cdot r_{1}^{e_{k+1}} \cdot r_{2}^{e_{k}} \cdot r_{3}^{e_{k-1}} \cdot r_{1} \\
& =a_{1} r_{1}^{e_{3 m-2+1}} r_{2}^{e_{3 m-2}} r_{3}^{e_{3 m-2-1}} \cdot r_{1} \\
& =a_{1} r_{1}^{m-1} r_{2}^{m-1} r_{3}^{m-1} \cdot r_{1} \\
& =a_{1} r_{1}^{\left\lfloor\frac{3 m}{3}\right\rfloor} r_{2}^{\left\lfloor m-1+\frac{2}{3}\right\rfloor} r_{3}^{\left\lfloor m-1+\frac{1}{3}\right\rfloor} \\
& =a_{1} r_{1}^{\left\lfloor\frac{(k+1)+1}{3}\right\rfloor} r_{2}^{\left\lfloor\frac{k+1}{3}\right\rfloor} r_{3}^{\left\lfloor\frac{(k+1)-1}{3}\right\rfloor}
\end{aligned}
$$

$\therefore \mathrm{P}(k+1)$ holds for $k=3 m-2$.

(ii.) If $k=3 m-1$, where $m \in N$, then $a_{k+1}=a_{k} \cdot r_{2}$

$$
\begin{aligned}
a_{k} & =a_{1} \cdot r_{1}^{e_{k+1}} \cdot r_{2}^{e_{k}} \cdot r_{3}^{e_{k-1}} \cdot r_{2} \\
& =a_{1} r_{1}^{e_{3 m-1+1}} r_{2}^{e_{3 m-1}} r_{3}^{e_{3 m-1-1}} \cdot r_{2} \\
& =a_{1} r_{1}^{m} r_{2}^{m-1} r_{3}^{m-1} \cdot r_{2} \\
& =a_{1} r_{1}^{\left\lfloor m+\frac{1}{3}\right\rfloor} r_{2}^{\left\lfloor\frac{3 m}{3}\right\rfloor} r_{3}^{\left\lfloor m-1+\frac{2}{3}\right\rfloor} \\
& =a_{1} r_{1}^{\left\lfloor\frac{(k+1)+1}{3}\right\rfloor} r_{2}^{\left\lfloor\frac{k+1}{3}\right\rfloor} r_{3}^{\left\lfloor\frac{(k+1)-1}{3}\right\rfloor}
\end{aligned}
$$

$\therefore \mathrm{P}(k+1)$ holds for $k=3 m-1$.

(iii.) If $k=3 m$, where $m \in N$, then $a_{k+1}=a_{k} \cdot r_{3}$

$$
\begin{aligned}
a_{k} & =a_{1} \cdot r_{1}^{e_{k+1}} \cdot r_{2}^{e_{k}} \cdot r_{3}^{e_{k-1}} \cdot r_{3} \\
& =a_{1} r_{1}^{e_{3 m+1}} r_{2}^{e_{3 m}} r_{3}^{e_{3 m-1}} \cdot r_{3} \\
& =a_{1} r_{1}^{m} r_{2}^{m} r_{3}^{m-1} \cdot r_{3} \\
& =a_{1} r_{1}^{\left\lfloor m+\frac{2}{3}\right\rfloor} r_{2}^{\left\lfloor m+\frac{1}{3}\right\rfloor} r_{3}^{\left\lfloor\frac{3 m}{3}\right\rfloor} \\
& =a_{1} r_{1}^{\left\lfloor\frac{(k+1)+1}{3}\right\rfloor} r_{2}^{\left\lfloor\frac{k+1}{3}\right\rfloor} r_{3}^{\left\lfloor\frac{(k+1)-1}{3}\right\rfloor}
\end{aligned}
$$

$\therefore \mathrm{P}(k+1)$ holds for $k=3 m$.

Therefore, (5) holds when $n=k+1$ and this proves the theorem.

Theorem 2.4. The formula of the general term of $a_{n}$ can also be

$$
a_{n}=a_{1} r^{e_{n-1}} r_{1}^{e_{n+1}-e_{n-1}} r_{2}^{e_{n}-e_{n-1}}
$$

where $r=r_{1} \cdot r_{2} \cdot r_{3}$ and $e_{i}=\left\lfloor\frac{i}{m}\right\rfloor$. 
The proof for theorem 2.4 is ommited but it can be easily verified using mathematical induction.

Theorem 2.5. The formula for the sum of the first $n$ terms of the sequence is given by

$$
\begin{gathered}
S_{n}=a_{1}\left(R\left(\frac{1-r^{e_{n-1}}}{1-r}\right)+1\right)+a_{1} r^{e_{n-1}}\left(r_{1}\left(\left\lfloor\frac{n+1}{3}\right\rfloor-\left\lfloor\frac{n}{3}\right\rfloor\right)\right) \\
+a_{1} r^{e_{n-1}}\left(\left(r_{1}+r_{1} r_{2}\right)\left(\left\lfloor\frac{n}{3}\right\rfloor-\left\lfloor\frac{n-1}{3}\right\rfloor\right)\right)
\end{gathered}
$$

where $R=r_{1}+r_{1} r_{2}+r_{1} r_{2} r_{3}, r=r_{1} r_{2} r_{3}$ and $e_{n-1}=\left\lfloor\frac{n-1}{3}\right\rfloor$

Proof. Let $p=e_{n-1}=\left\lfloor\frac{n-1}{3}\right\rfloor, R=r_{1}+r_{1} r_{2}+r_{1} r_{2} r_{3}$ and $r=r_{1} r_{2} r_{3}$.

$$
\begin{aligned}
S_{n}= & a_{1}+a_{1} r_{1}+a_{1} r_{1} r_{2}+a_{1} r_{1} r_{2} r_{3}+a_{1} r_{1}^{2} r_{2} r_{3}+a_{1} r_{1}^{2} r_{2}^{2} r_{3}+a_{1} r_{1}^{2} r_{2}^{2} r_{3}^{2} \\
& +a_{1} r_{1}^{3} r_{2}^{2} r_{3}^{2}+a_{1} r_{1}^{3} r_{2}^{3} r_{3}^{2}+a_{1} r_{1}^{3} r_{2}^{3} r_{3}^{3}+\ldots+a_{1} r_{1}^{e_{n-1}} r_{2}^{e_{n-2}} r_{3}^{e_{n-3}} \\
& +a_{1} r_{1}^{e_{n}} r_{2}^{e_{n-1}} r_{3}^{e_{n-2}}+a_{1} r_{1}^{e_{n+1}} r_{2}^{e_{n}} r_{3}^{e_{n-1}} \\
= & a_{1}+a_{1} R+a_{1} r R+a_{1} r^{2} R+\ldots+a_{1} r^{p-1} R+a_{1} r_{1}^{e_{n}} r_{2}^{e_{n-1}} r_{3}^{e_{n-2}} \\
& +a_{1} r_{1}^{e_{n}+1} r_{2}^{e_{n}} r_{3}^{e_{n-1}} \\
= & a_{1}+a_{1} R\left(1+r+r^{2}+\ldots+r^{p-1}\right)+a_{1} r_{1} r^{p}\left(\left\lfloor\frac{n+1}{3}\right\rfloor-\left\lfloor\frac{n}{3}\right\rfloor\right) \\
& +a_{1} r^{p}\left(r_{1}+r_{1} r_{2}\right)\left(\left\lfloor\frac{n}{3}\right\rfloor-\left\lfloor\frac{n-1}{3}\right\rfloor\right) \\
= & a_{1}+a_{1} R\left(\frac{1-r^{p}}{1-r}\right)+a_{1} r_{1} r^{p}\left(\left\lfloor\frac{n+1}{3}\right\rfloor-\left\lfloor\frac{n}{3}\right\rfloor\right) \\
& +a_{1} r^{p}\left(r_{1}+r_{1} r_{2}\right)\left(\left\lfloor\frac{n}{3}\right\rfloor-\left\lfloor\frac{n-1}{3}\right\rfloor\right)
\end{aligned}
$$

\section{References}

[1] Zhang Xiong and Zhang Yilin, "Sequence of numbers with alternate common differences", Scientia Magna, High American Press Vol.3, No.1, (2007), pp.93-97. 